Brief report

\title{
Preliminary data on immunogenicity, safety and tolerability of trivalent inactivated influenza vaccine in children with inborn errors of metabolism at risk of decompensation
}

\author{
Q1 Susanna Esposito ${ }^{\mathrm{a}, *}$, Filippo Salvini ${ }^{\mathrm{b}}$, Francesca Menni ${ }^{\mathrm{a}}$, Alessia Scala ${ }^{\mathrm{a}}$, \\ Elisabetta Salvatici ${ }^{\mathrm{b}}$, Francesca Manzoni ${ }^{\mathrm{a}}$, Enrica Riva ${ }^{\mathrm{b}}$, Marcello Giovannini $^{\mathrm{a}}$, \\ Nicola Principi ${ }^{a}$ \\ a Pediatric Clinic 1, Department of Pathophysiology and Transplantation, Università degli Studi di Milano, Fondazione IRCCS Ca' Granda Ospedale Maggiore \\ Policlinico, Milan, Italy \\ ${ }^{\mathrm{b}}$ Pediatric Clinic, San Paolo Hospital, Università degli Studi di Milano, Milan, Italy
}

\section{A R T I C L E I N F O}

\section{Article history:}

Received 16 May 2013

Received in revised form 12 August 2013

Accepted 21 August 2013

Available online $\mathrm{xxx}$

\section{Keywords:}

Children

Influenza virus

Influenza vaccine

Metabolic disease

Trivalent influenza vaccine

\begin{abstract}
A B S T R A C T
In order to evaluate the immunogenicity, safety and tolerability of influenza vaccination in children with inborn errors of metabolism (IEMs), we enrolled 20 patients with IEMs at risk of decompensation (14 males; mean age \pm SD, $8.5 \pm 3.9$ years) and 20 healthy age- and gender-matched controls. Four weeks after vaccination, seroconversion rates were $75-85 \%$ and seroprotection rates $85-95 \%$, with high geometric mean titers (GMTs) of all three influenza antigen strains in both groups. Three months after vaccination, most of the subjects remained seroconverted with high seroprotection rates and high GMTs for all the three influenza strains. Safety and tolerability were also very good, with no differences between the groups.
\end{abstract}

(C) 2013 Published by Elsevier Ltd.

\section{Introduction}

The patients for whom influenza vaccination is recommended by all health authorities includes those with inborn errors of metabolism (IEMs) [1]. The main reason for this is that IEMs include diseases that are at high risk of metabolic decompensation after the onset of a febrile infectious disease [2] Moreover, some IEMs are associated with a severe underlying disease (such as neurological involvement or concomitant immunodeficiency) that may lead to influenza-related complications [3]. However, preventing influenza in such children is not as simple as in healthy subjects because the administration of vaccine can be followed by adverse events including fever, and this may worsen their fragile metabolic equilibrium [4]. Furthermore, influenza vaccine may be less effective because many IEMs are accompanied by an inefficient immune system [5].

\footnotetext{
* Corresponding author at: Pediatric Clinic 1, Department of Pathophysiology and Transplantation, Università degli Studi di Milano, Fondazione IRCCS Ca' Granda Ospedale Maggiore Policlinico, Via Commenda 9, 20122 Milano, Italy. Tel.: +39 02 55032498; fax: +390250320206.

E-mail address: susanna.esposito@unimi.it (S. Esposito).
}

It has recently been pointed that, although official recommendations strongly suggest that patients with IEMs should receive routine vaccinations using the same schedule as that used for healthy subjects, we know very little about the immunogenicity, safety and tolerability of many vaccines in patients with IEMs [6,7]. Consequently, studies regarding the effect of the different vaccines in children with IEMs are strongly advocated, particularly in those at high risk of decompensation.

The aim of this study was to collect data concerning the immunogenicity, safety and tolerability of a trivalent inactivated influenza vaccine (TIV) in children with IEMs at risk of decompensation.

\section{Materials and methods}

\subsection{Study population}

The study involved 20 children aged 3-18 years (14 males; mean age \pm SD $8.5 \pm 3.9$ years) with IEM at risk of decompensation according to the definition of Kingsley et al. (10 amino acid disorders, seven cases of glycogen storage disease type I, and three of methylmalonic aciduria) [7], who had been clinically stable for at least three months and were regularly attending the pediatric 
60 outpatient clinic of the Fondazione IRCCS Ca' Granda Ospedale Maggiore Policlinico or the San Paolo Hospital of the University of Milan (Italy). A total of 20 age- and sex-matched healthy children (14 males; mean age $\pm \mathrm{SD}, 8.1 \pm 3.3$ years) were enrolled as controls. All of the patients and controls had been vaccinated against influenza in the previous three seasons.

The study protocol was approved by the Ethics Committee of the Fondazione IRCCS Ca' Granda Ospedale Maggiore Policlinico and San Paolo Hospital, Milan, Italy. Written informed consent to participate in the study was obtained from all of the subjects aged $\geq 7$ years, and from the parents or legal guardians.

\subsection{Study procedures}

All of the subjects received a single dose of a TIV (Fluarix, GlaxoSmithKline Biologicals, Rixensart, Belgium) by means of an injection in the deltoid muscle in November 2012. The vaccine was formulated in accordance with the World Health Organization recommendations for the 2012-2013 northern hemisphere influenza season. Each dose consisted of $15 \mu \mathrm{g}$ each of purified A/California/7/2009(H1N1)pdm09-like, A/Victoria/361/2011(H3N2)-derived and B/Wisconsin/1/2010(B)like influenza surface antigen hemagglutinin, with solvent added to reach $0.5 \mathrm{~mL}$. Serum samples were collected for antibody assay immediately before the vaccine was administered, and four weeks ( $28 \pm 3$ days) and three months ( $90 \pm 3$ days) later.

The subjects were observed for $30 \mathrm{~min}$ after the injection, and their parents recorded the occurrence of solicited and unsolicited local symptoms (erythema, swelling/induration, and pain) or systemic symptoms (an axillary temperature of $\geq 38^{\circ} \mathrm{C}$, irritability, sleepiness, changes in eating habits, vomiting, diarrhea, malaise, muscle ache) for the next 14 days. The symptoms were considered mild if they did not interfere with normal everyday activities, and severe if they prevented them and required medical attention. Adverse reactions were defined as any reaction that persisted for longer than seven days after the vaccination, and serious adverse reactions as any reaction that required medical attention or hospitalisation during the study period.

\subsection{Laboratory assays}

Immunogenicity was evaluated by means of a standard assay of hemagglutination-inhibiting ( $\mathrm{HI}$ ) antibodies to the influenza strains contained in the vaccine [8]. The serum samples were tested in duplicate at an initial dilution of $1: 10$, and those that were negative for the antibody were assigned an arbitrary titer of 1:5. HI antibody titers were expressed as the reciprocal of the highest serum dilution that completely inhibited hemagglutination. In accordance with the criteria described in the European Medicines Agency (EMA) guideline [9], humoral immune response was assessed on the basis of the seroconversion rate (defined as the percentage of subjects experiencing at least a 4 -fold increase in a seropositive pre-vaccination $\mathrm{HI}$ antibody titer, or an increase from $<10$ to $\geq 40$ in those who were seronegative), the seroprotection rate (defined as the percentage of subjects reaching an HI titer of $\geq 40$ ), and geometric mean titers (GMTs).

\section{$112 \quad$ 2.4. Statistical analysis}

The continuous variables are given as mean values \pm standard deviation (SD), and the categorical variables as numbers and percentages. The continuous data were analyzed using a two-sided Student's test if they were normally distributed (on the basis of the Shapiro-Wilk statistic), or a two-sided Wilcoxon rank-sum test if they were not. The categorical data were analyzed using contingency table analysis and the chi-squared or Fisher's test, as appropriate. All of the analyses were two-tailed, and $p$ values of 0.05 or less were considered significant.

\section{Results}

Table 1 shows the immune responses of the two groups after the administration of the vaccine. More than $50 \%$ of the patients and controls had baseline specific antibody titers $\geq 40$ upon HI assay against $\mathrm{A} / \mathrm{H} 1 \mathrm{~N} 1$ and $\mathrm{A} / \mathrm{H} 3 \mathrm{~N} 2$ strains, and about $50 \%$ of the patients and $40 \%$ of the controls had baseline specific antibody titers of $\geq 40$ upon HI assay against the B strain. Four weeks after the administration of the vaccine, the patients and controls respectively had seroconversion rates of $85 \%$ and $75 \%$ against $\mathrm{A} / \mathrm{H} 1 \mathrm{~N} 1,75 \%$ and $85 \%$ against $\mathrm{A} / \mathrm{H} 3 \mathrm{~N} 2$, and $75 \%$ and $80 \%$ against the $B$ strain, with no significant difference between the groups. The seroprotection rates were respectively $95 \%$ and $85 \%$ against $\mathrm{A} / \mathrm{H} 1 \mathrm{~N} 1,90 \%$ and $95 \%$ against $\mathrm{A} / \mathrm{H} 3 \mathrm{~N} 2$, and $90 \%$ and $85 \%$ against $B$ strain, once again with no significant between-group difference. GMTs and the increase in antibody levels were similarly high in both groups.

Three months after the vaccination, most of the subjects remained seroconverted and were still seroprotected, without any statistically significant difference between the groups. Their GMTs and the increase from baseline remained similarly high in both groups. There were no differences between the patients with different IEM's.

Table 2 summarizes the incidence of local and systemic reactions in the two groups during the 14 days after vaccination. In both groups, $15 \%$ of the subjects experienced local reactions and $25 \%$ systemic reactions. The distribution of the adverse reactions was similar in the two groups, and there were no serious adverse events.

Table 1

Immune responses to a trivalent influenza vaccine in children with inborn errors of metabolism (IEMs) at risk of decompensation and healthy controls.

\begin{tabular}{|c|c|c|}
\hline Immune response & $\begin{array}{l}\text { Children with IEMs } \\
(n=20)\end{array}$ & $\begin{array}{l}\text { Healthy controls } \\
(n=20)\end{array}$ \\
\hline \multicolumn{3}{|l|}{ A/H1N1 } \\
\hline \multicolumn{3}{|l|}{ Seroconversion, No. (\%) } \\
\hline After $28 \pm 3$ days & $17(85.0)$ & $15(75.0)$ \\
\hline After $90 \pm 3$ days & $14(70.0)$ & $13(65.0)$ \\
\hline \multicolumn{3}{|l|}{ Seroprotection, No. (\%) } \\
\hline After $28 \pm 3$ days & $19(95.0)$ & $17(85.0)$ \\
\hline After $90 \pm 3$ days & $19(95.0)$ & $17(85.0)$ \\
\hline GMT, baseline & 78.3 & 103.6 \\
\hline After $28 \pm 3$ days (fold increase) & $939.6(12)$ & $864.9(8.3)$ \\
\hline After $90 \pm 3$ days (fold increase) & $464.0(5.9)$ & $376.1(3.6)$ \\
\hline \multicolumn{3}{|l|}{ A/H3N2 } \\
\hline \multicolumn{3}{|l|}{ Seroconversion, No. (\%) } \\
\hline After $28 \pm 3$ days & $15(75.0)$ & $15(85.0)$ \\
\hline After $90 \pm 3$ days & $13(65.0)$ & $13(65.0)$ \\
\hline \multicolumn{3}{|l|}{ Seroprotection, No. (\%) } \\
\hline After $28 \pm 3$ days & $18(90.0)$ & $19(95.0)$ \\
\hline After $90 \pm 3$ days & $17(85.0)$ & $18(90.0)$ \\
\hline GMT, baseline & 53.1 & 74.9 \\
\hline After $28 \pm 3$ days (fold increase) & $373.0(7.0)$ & $457.4(6.1)$ \\
\hline After $90 \pm 3$ days (fold increase) & $284.5(5.4)$ & $373.3(4.9)$ \\
\hline \multicolumn{3}{|l|}{ B } \\
\hline \multicolumn{3}{|l|}{ Seroconversion, No. (\%) } \\
\hline After $28 \pm 3$ days & $15(75.0)$ & $16(80.0)$ \\
\hline After $90 \pm 3$ days & $13(65.0)$ & $14(70.0)$ \\
\hline \multicolumn{3}{|l|}{ Seroprotection, No. (\%) } \\
\hline After $28 \pm 3$ days & $18(90.0)$ & $17(85.0)$ \\
\hline After $90 \pm 3$ days & $18(90.0)$ & $17(85.0)$ \\
\hline GMT, baseline & 43.8 & 30.5 \\
\hline After $28 \pm 3$ days (fold increase) & $587.8(13.4)$ & $471.5(15.5)$ \\
\hline After $90 \pm 3$ days (fold increase) & $376(8.6)$ & $371.3(12.2)$ \\
\hline
\end{tabular}

GMT: geometric mean titer. There were no significant differences between the two groups. 
Table 2

Summary of local and systemic reactions in the 14 days following vaccination with a trivalent influenza vaccine in children with inborn errors of metabolism (IEMs) at risk of decompensation and healthy controls.

\begin{tabular}{lll}
\hline Adverse events & $\begin{array}{l}\text { Children with IEMs } \\
(n=20)\end{array}$ & $\begin{array}{l}\text { Healthy controls } \\
(n=20)\end{array}$ \\
\hline $\begin{array}{l}\text { Local reactions, No. (\%) } \\
\text { Erythema }\end{array}$ & $1(5.0)$ & $1(5.0)$ \\
$\quad$ Swelling/induration & $1(5.0)$ & $1(5.0)$ \\
$\quad$ Pain & $2(10.0)$ & $1(5.0)$ \\
At least one local event & $3(15.0)$ & $3(15.0)$ \\
Systemic reactions, No. (\%) & & \\
Fever $\geq 38^{\circ} \mathrm{C}$ & $3(15.0)$ & $2(10.0)$ \\
Rhinitis & $1(5.0)$ & $2(10.0)$ \\
Malaise & $2(10.0)$ & $2(10.0)$ \\
Spleepiness & $1(5.0)$ & $1(5.0)$ \\
$\quad$ Changed eating habits & $2(10.0)$ & $1(5.0)$ \\
$\quad$ Vomiting & $1(5.0)$ & $0(0.0)$ \\
Diarrhea & $1(5.0)$ & $5(25.0)$ \\
At least one systemic event & $5(25.0)$ & $6(30.0)$ \\
At least one local or systemic event & $6(30.0)$ & $4(20.0)$ \\
Drugs required for local or systemic & $5(25.0)$ & $0(0.0)$ \\
events & & \\
Serious adverse events & $0(0.0)$ & \\
\hline
\end{tabular}

There were no significant differences between the two groups.

During the study period, no influenza-like illness was reported in either group, and no metabolic crisis was reported in the patients with IEMs.

\section{Discussion}

Our findings indicate that the administration of a TIV seems to evoke an antibody response in children with IEMs potentially associated with immune system deficiency that is no different from that observed in healthy subjects. Most of the enrolled patients had IEMs that have been associated with immune deficiency, such as amino acid disorders (which have been related to cellular immune defects in number and function, and decreased IgG and IgA levels) $[10,11]$ and methylmalonic aciduria (in which neutropenia, impaired phagocytic chemotaxis, low B and T lymphocyte counts and low IgG levels have all been identified as individual defects in different patients) [12]. However, it has been repeatedly reported that the immunological abnormalities disappear or are significantly reduced when the metabolic defect is corrected because the main cause of the reduced immune activity seems to be the inborn error itself, which may block a metabolic process that is essential for immune function or produce a toxic metabolite that impairs the immune system $[1,6,7]$. This may explain why our patients (all of whom were clinically stable) showed an adequate immune response to TIV that was similar to that observed in the healthy controls.

Moreover, although the patients with IEMs were theoretically at risk of metabolic decompensation, TIV was safe and well tolerated, and there was no difference in the incidence of adverse events between them and the controls. This confirms that the negative effects of vaccines rarely occur in children with stable IEMs but mainly appear in children with unstable metabolic conditions.
Children who have been clinically stable for a long time can receive TIV without any increase in the risk of adverse events.

Although these data are preliminary and need to be confirmed in larger study populations, they could be extrapolated when considering the use of TIV use in subjects with IEMs at risk of decompensation other than those included in this study. Given the higher risk of influenza-related complications in children with IEMs, our findings also support the recommendation that they should receive annual influenza vaccinations [7]. Further studies carried in naïve subjects with IEM as well as in children with different metabolic conditions and more pronounced immune deficits will define the best way of using TIV in order to obtain the maximum benefit and establish whether the recommendation should be refined on the basis of the status of the immune system or the risk of catabolic events.

\section{Conflict of interest}

None of the authors has any commercial or other relationship that might pose a conflict of interest.

\section{Acknowledgements}

This study was supported in part by a grant from the Italian Ministry of Health (Bando Giovani Ricercatori 2007). Appropriate informed consent was obtained, and the study was carried out in accordance with the guidelines for human experimentation specified by the authors' institutions.

\section{References}

[1] Menni F, Chiarelli G, Sabatini C, Principi N, Esposito S. Vaccination in children with inborn errors of metabolism. Vaccine 2012;30:7161-4.

[2] Varghese M, Cafferkey M, O'Regan M, Monavari AA, Treacy EP. Should children with inherited metabolic disorders receive varicella vaccination? Arch Dis Child 2011;96:99-100.

[3] Ming JE, Stiehm ER, Graham Jr JM. Syndromic immunodeficiencies: genetic syndromes associated with immune abnormalities. Crit Rev Clin Lab Sci 2003;40:587-642.

[4] Yang Y, Sujan S, Sun F, Zhang Y, Jiang Y, Song J, et al. Acute metabolic crisis induced by vaccination in seven Chinese patients. Pediatr Neurol 2006;35:114-8.

[5] Klein NP, Aukes L, Lee J, Fireman B, Shapira SK, Slade B, et al. Evaluation of immunization rates and safety among children with inborn errors of metabolism. Pediatrics 2011;127:e1139-46.

[6] Brady MT. Immunization recommendations for children with metabolic disorders: more data would help. Pediatrics 2006;118:810-3.

[7] Kingsley JD, Varman M, Chatterjee A, Kingsley RA, Roth KS. Immunizations for patients with metabolic disorders. Pediatrics 2006;118:e460-70.

[8] Menegon T, Baldo V, Bonello C, Dalla Costa D, di Tommaso D, Trivello R. Influenza vaccines: antibody responses to split virus and MF59-adjuvanted subunit virus in an adult population. Eur J Epidemiol 1999;15:573-6.

[9] The European Agency for the Evaluation of Medicinal Products (EMEA); Committee for Proprietary Medicinal Products (CPMP). Note for guidance on harmonisation of requirements for influenza vaccines (CPMP/BWP/214/96), London, March 12; 1997.

[10] Becroft DM, Phillips LI, Webster DR, Wilson JD. Absence of immune deficiency in hereditary orotic aciduria. N Engl J Med 1984;310:1333-4.

[11] Lukkarinen M, Parto K, Ruuskanen O, Vainio O, Käyhty H, Olander RM, et al. T cell immunity in patients with lysinuric protein intolerance. Clin Exp Immunol 1999;116:430-4.

[12] Wong SN, Low LC, Lau YL, Nicholls J, Chan MY. Immunodeficiency in methylmalonic acidaemia. J Paediatr Child Health 1992;28:180-3. 